\title{
Translating immune targets into treatments for kidney disease
}

Immune mechanisms contribute substantially to the development of kidney disease. Autoimmune processes can have direct deleterious effects on the kidney, as seen in membranous nephropathy and antiglomerular basement membrane glomerulonephritis. Systemic autoimmune conditions, such as systemic lupus erythematosus and anti-neutrophil cytoplasmic antibody-associated vasculitis, can also lead to kidney injury (Holdsworth, S. R. et al. Nat. Rev. Nephrol. http://dx.doi.org/10.1038/nrneph.2016.18). Moreover, inflammation can exacerbate organ damage and contribute to the progression of non-autoimmune forms of kidney disease (Meng, X.-M. et al. Nat. Rev. Nephrol. 10, 493-503, 2014; Jang, H. R. \& Rabb, H. Nat. Rev. Nephrol. 11, 88-101, 2015). Finally, immune responses are a key determinant of the success of kidney transplantation. Together these effects highlight immune function as a multifaceted determinant of renal health and underscore the importance of the immune system as a key target for the development of therapeutics for kidney disease.

The development of agents to target specific immunologic processes is not a new area of research; much progress has been made in the development of targeted treatments for autoimmune and inflammatory conditions such as rheumatoid arthritis and inflammatory bowel disease. Despite these advances and improved understanding of the importance of immune function in the pathogenesis of kidney disease, relatively few agents that target specific immunologic processes have been successfully added to the therapeutic armamentarium for patients with immune-mediated kidney disease. In response to this lack of progress, in April 2016 the International Society of Nephrology will hold a Nexus symposium on 'translational immunology in kidney disease, to promote discussion and collaboration with the aim of identifying ways to facilitate the translation of new immunologic targets, identified through basic and preclinical research, into novel therapies. This focus issue of Nature Reviews Nephrology features four Reviews written by key opinion leaders who will present at this symposium, and highlights the current progress and remaining challenges in the translation of immunologic therapies for kidney disease.

The first Review in this issue directly addresses possible reasons for the lack of progress in the development of immunologic therapies for immune-mediated kidney disease. Hans-Joachim Anders et al. argue that the problem is not that appropriate drug targets have not been found, but rather, that the approaches that have been used to identify targets and measure outcomes are flawed. They describe seven hurdles to the development and introduction of new immunologic therapies for immune-mediated kidney disease, including fundamental issues relating to the definition of disease categories, the identification of appropriate study end points, use of appropriate trial design, and the dearth of available approaches to accurately identify organ injury.

Biologic agents have substantially advanced the treatment of immunological disorders, including chronic inflammatory and autoimmune diseases. In their Review, Stephen Holdsworth and colleagues provide an overview of the major immunopathogenic pathways that contribute to the development of autoimmune renal disease and describe the potential use of biologics to specifically target these pathways. The increasing availability of biologics will hopefully lead to advances in the treatment of autoimmune renal disease; however, alternative approaches must also be explored to optimize the chances of success. In this issue, Falk Hiepe and Andreas Radbruch describe how the selective depletion of long-lived antibody-secreting autoreactive plasma cells in an autoantigen-specific manner targets pathogenic humoral memory and could potentially improve outcomes for patients with autoimmune renal disease. In a separate Review, Federica Casiraghi et al. discuss the potential of bone marrow-derived multipotent mesenchymal stromal cells (MSCs) to induce tolerance by altering the balance between effector and regulatory pathways. They describe findings from preclinical and initial clinical transplantation studies and discuss the remaining challenges, including unanswered questions regarding the risks and potential benefits of MSCs as a candidate for cell therapy in organ transplantation.

Together these Reviews demonstrate that these are exciting times in drug development, but importantly, the authors all acknowledge that considerable challenges remain in translating advances in target identification into the development of new agents. Addressing these challenges will require improvements to preclinical and clinical study design, the use of multicentre international cohorts, and approaches to improve the characterization of specific disease entities and monitor disease progression. We hope that this focus issue stimulates discussion as to how these aims can be achieved with the ultimate goal of improving outcomes for patients with immune-mediated renal disease. 